\title{
Steady State Analysis of Markov Regenerative SPN with Age Memory Policy
}

\author{
Miklós Telek ${ }^{1}$, Andrea Bobbio ${ }^{2}$, László Jereb ${ }^{1}$ \\ Antonio Puliafito ${ }^{3}$, Kishor S. Trivedi ${ }^{4}$ \\ 1 Department of Telecommunications \\ Technical University of Budapest, \\ 1521 Budapest, Hungary \\ ${ }^{2}$ Dipartimento di Elettronica per l'Automazione \\ Università di Brescia, 25123 Brescia, Italy \\ ${ }^{3}$ Istituto di Informatica e Telecomunicazioni \\ Università di Catania, 95125 Catania, Italy \\ ${ }^{4}$ Department of Electrical Engineering \\ Duke University, Durham NC 27708-0291, USA
}

\begin{abstract}
Non-Markovian models represent a powerful tool to deal with systems characterized by non exponentially distributed timed events. Recently, some effort has been devoted to the study of Petri nets with generally distributed events, whose underlying marking process belongs to the class of Markov Regenerative Processes (MRGP). In this paper, we describe a computationally effective algorithm for the steady state solution of MRSPN with age memory policy and subordinated Continuos Time Markov Chain (CTMC).
\end{abstract}

Key words: Stochastic Petri Nets, Generally distributed transitions, Markov regenerative processes, Preemptive resume policies.

\section{Introduction}

In the last decade several classes of Stochastic Petri Nets (SPNs) have been developed to deal with non-exponentially distributed events $[9,4]$. The main reason for this is the observation that it is possible to identify a wide number of real situations in which deterministic or generally distributed events occur. Events such as timeouts in a protocol, service times in a manufacturing system performing the same task on each part, and memory access or instruction execution in a low-level $\mathrm{h} / \mathrm{w}$ or $\mathrm{s} / \mathrm{w}$ model, have durations which are constant or with a very low variance. Similarly, block request size to an I/O subsystem have an unusual distribution that cannot be properly captured by an exponential. Phase-type distributions can be used to approximate the occurrence time of a generally distributed event, but the main drawback of this approach is in the often prohibitive state space size of the underlying stochastic process.

The analysis of stochastic systems with non-exponential timing is of increasing interest in the literature and requires the development of suitable modeling tools. In [8] Markov Regenerative Stochastic Processes (MRGPs) have been proved to be an effective way to capture the 
behavior of the stochastic process underlying a $S P N$ where at most one generally distributed transition is enabled in each marking. This class of $S P N$ has been named as $M R S P N^{*}$. The solution proposed in [8] is based on the derivation of the time-dependent transition probability matrix in the Laplace transform domain, followed by a numerical inversion. German and Lindemann [14] have proposed to derive the steady state solution of the same model by resorting to the method of supplementary variables [11]. The possibility of applying the methodology of supplementary variables to the transient analysis of $D S P N$ s is explored in [13], where, however, only very special cases are taken into consideration. To remove the limitation that at most one generally distributed transition can be enabled in a given marking, in [7] the transient and steady-state behavior of an SPN with many generally distributed transitions enabled at the same time is provided, under some structural restrictions.

Even if a very fertile line of research has been opened by recognizing the underlying stochastic process of a $M R S P N$ to be an $M R G P$ (many interesting applications and improvements can be found in [17]), the main limitation of the models discussed in the previous references is that the generally distributed (or deterministic) transitions must be assigned a firing policy of enabling memory type.

A more realistic assumption is to adopt an age memory policy, according to which the amount of work already done by a running activity is not lost if the activity is temporarily suspended. Many pratical situations behave according to this modeling extension. Consider for example, a fault tolerant, parallel computing system, where a single task may be interrupted either during a fault/recovery cycle or for the execution of a higher priority task, but when the cause originating the interruption is ceased, the dormant task is resumed from the point its interruption occurred. The enabling memory policy is not able to capture this behavior.

To overcome the previous restriction, Bobbio and Telek have introduced the class of AgeD$S P N$ [5], in which a transition can be associated an age memory policy. In [6] they further extended the previous results by defining the class of $M R S P N$ with non-overlapping activity cycles. The main characterization of this class is that multiple generally distributed transitions can be simultaneously enabled, provided that a dominant transition exists, whose enabling period determines the occurrence of two successive regeneration time points.

In this paper, we propose a computationally effective approach to the steady state analysis of the class of MRSPN with non-overlapping activity cycles and assuming that the subordinated process to any regeneration period is a $C T M C$. The proposed method contains the existing results for the steady state analysis of $D S P N$ and $M R S P N^{*}$ as special cases.

The paper is organized as follows. Section 2 provides a formal definition of Generally Distributed Transition_SPN (GDT_SPN), and discusses the different memory policies. Section 3 defines a $M R S P N$ with non-overlapping activity cycles. In Section 4 a unified transient analysis of MRSPN with non overlapping activity cycles is presented. Section 5 focuses on the steady state analysis when the subordinated process is a CTMC. Section 6 illustrates the computational method for the steady-state analysis and discusses its complexity. Finally, Section 7 presents the steady-state solution of a preempitive $M / D / 1 / n+m / n+m$ queueing system with two classes of customers.

\section{Markov Regenerative Stochastic Petri Nets}

A standard technique for the probabilistic study of a Petri net is to examine the marking process, $\{\mathcal{M}(t), t>0\}$, obtained by constructing the reachability graph for the net. Once the Reachability Set $(\mathcal{R S})$ of the net is identified, namely the set of all possible states (markings) 
of the system, the reachability graph $(\mathcal{R G})$ can be obtained by connecting a marking $M_{i}$ to a marking $M_{j}$ with a directed arc if the marking $M_{j}$ can result from the firing of some transition enabled in $M_{i}$. From the given initial marking $M_{0}$, a unique reachability graph is obtained. If the Petri net model comprises both timed and immediate transitions, $\mathcal{R S}\left(M_{0}\right)$ is partitioned into tangible (no immediate transitions are enabled) and vanishing markings. As the time spent by the marking process in the latter is zero, since they enable at least one immediate transition, they can be eliminated from a study of the marking process as they have no effect on the state probabilities [2]. We thus obtain a reduced reachability graph in which the vanishing states are no longer present explicitly, but only in virtue of their modification of the passage rate between tangible states. Thus, in the following, we implicitly assume that, whenever it is the case, all the vanishing markings have already been removed from the original reachability graph.

The following is a formal definition of Generally Distributed Transition_SPN (GDT_SPN), in which we allow for non-exponentially distributed timed transitions. Memory variables are introduced to record the amount of time a transition has been enabled. The definition is general enough to include the $D S P N$ as well as $M R S P N^{*}$ classes. Further it allows for different memory policies to be implemented.

Definition 1 - A GDT_SPN is a marked $S P N$ in which [1]:

- To any timed transition $t r_{k} \in T$ is associated a random variable $\gamma_{k}$ modeling the time needed by the activity represented by $t_{k}$ to complete, when considered in isolation.

- Each random variable $\gamma_{k}$ is characterized by its cumulative distribution function $G_{k}(t)$.

- To each timed transition $\operatorname{tr}_{k}$ is associated a memory variable $a_{k}$ and a memory policy; the memory policy specifies the functional dependence of the memory variable on the past enabling time of the transition.

- A initial probability is given on the reachability set.

The memory variable $a_{k}$, associated to transition $t r_{k}$, is a functional that depends on the time during which $\operatorname{tr}_{k}$ has been enabled. The memory variables together with their memory policies uniquely specify how the underlying stochastic process is conditioned upon its past history. The semantics of different memory policies has been discussed in [1] where three alternatives have been proposed and examined.

- Resampling policy - The memory variable $a_{k}$ is reset to zero at any change of marking.

- Enabling memory policy - The memory variable $a_{k}$ accounts for the work performed by the activity corresponding to $t r_{k}$ from the last epoch in which $t r_{k}$ has been enabled. When transition $t r_{k}$ is disabled (even without firing) the corresponding enabling memory variable is reset.

- Age memory policy - The memory variable $a_{k}$ accounts for the work performed by the activity corresponding to $t r_{k}$ from its last firing up to the current epoch and is reset only when $t r_{k}$ fires.

At the entrance in a new tangible marking, the residual firing time is computed for each enabled timed transition given its memory variable, so that the next marking is determined by the minimal residual firing time among the enabled transitions (race policy [1]). Because of the memoryless property, the value of the memory variable is irrelevant in determining the residual firing time for exponential transitions, and the three mentioned policies are completely 
equivalent. Hence, for an exponential transition $t r_{k}$, we assume, conventionally, that the corresponding memory variable is always identically zero. We can therefore partition the set of the transitions into EXP transitions with associated an exponential r.v. and identically zero memory variable, and GEN transition with associated any r.v. (including the deterministic case) and memory variable increasing in the enabling markings.

Definition 1 allows for generally distributed transitions to be included in a $P N$. The next step is to characterize the underlying stochastic process in such a way that it remains analytically tractable. The technique usually adopted is to identify some time points at which it is possible to forget the past history of the process. These points, indicated as regeneration points, are such that the future evolution of the stochastic process only depends on the present state entered when a regeneration time point occurs.

A single realization of the marking process $\mathcal{M}(t)$ can be written as:

$$
\mathcal{R}=\left\{\left(\tau_{0}, M_{0}\right) ;\left(\tau_{1}, M_{1}\right) ; \ldots ;\left(\tau_{i}, M_{i}\right) ; \ldots\right\}
$$

where $M_{i+1}$ is a marking immediately reachable from $M_{i}$, and $\tau_{i+1}-\tau_{i}$ is the sojourn time in marking $M_{i}$. With the above notation, $\mathcal{M}(t)=M_{i}$ for $\tau_{i} \leq t<\tau_{i+1}$.

If at time $\tau_{i}^{+}$of entrance in a tangible marking $M_{i}$ all the memory variables $a_{k}(k=$ $\left.1,2, \ldots, n_{t}\right)$ are equal to zero, we say that $\tau_{i}$ is a regeneration time point for the marking process $\mathcal{M}(t)$. Let us denote by $\tau_{n}^{*}$ the sequence of the regeneration time points embedded into a realization $\mathcal{R}$. The tangible marking $M_{n}$ entered at a regeneration time point $\tau_{n}^{*}$ is called a regeneration marking.

The following proposition provides a necessary condition for a time point to be a regeneration point.

Proposition 1 A regeneration time point $\tau_{n}^{*}$ in the marking process $\mathcal{M}(t)$ is the epoch of entrance in a tangible marking $M_{n}$ in which all the memory variables are equal to 0.

The embedded sequence of regeneration time points $\left(\tau_{n}^{*}, M_{n}\right)$ is a Markov renewal sequence and the marking process $\mathcal{M}(t)$ is a Markov regenerative process $[10,8,9]$ (also known as semi-regenerative process).

Definition 2 - $A$ GDT_SPN, for which an embedded Markov renewal sequence $\left(\tau_{n}^{*}, M_{n}\right)$ exists, is a Markov Regenerative Stochastic Petri Nets (MRSPN) [8].

Formally this implies that, since $\left(\tau_{n}^{*}, M_{n}\right)$ is a Markov renewal sequence, the following equalities hold:

$$
\begin{aligned}
& \operatorname{Pr}\left\{M_{n+1}=j,\left(\tau_{n+1}^{*}-\tau_{n}^{*}\right) \leq t \mid M_{n}=i, \tau_{n}^{*}, M_{n-1}, \tau_{n-1}^{*}, \ldots, M_{(0)}, \tau_{0}^{*}\right\}= \\
& \operatorname{Pr}\left\{M_{n+1}=j,\left(\tau_{n+1}^{*}-\tau_{n}^{*}\right) \leq t \mid M_{n}=i, \tau_{n}^{*}\right\}= \\
& \operatorname{Pr}\left\{M_{1}=j, \tau_{1}^{*} \leq t \mid M_{0}=i\right\}
\end{aligned}
$$

The first equality expresses the Markov property (i.e., in any regeneration time point the condition on the past is condensed in the present state). The second equality expresses the time homogeneity (i.e. the probability measures are independent of a translation along the 
time axis), due to the fact that the semantic of a $G D T_{-} S P N$ and the firing distribution of the transitions do not change with time.

To provide an analytical formulation of the stochastic process underlying a GDT_SPN, according to $[8,10]$, we define the following matrix valued functions (of dimension $n \times n$ ), where $n$ is the cardinality of the tangible subset of $\mathcal{R S}\left(M_{0}\right)$ :

$$
\begin{aligned}
& \mathbf{V}(t)=\left[V_{i j}(t)\right] \text { such that } V_{i j}(t)=\operatorname{Pr}\left\{\mathcal{M}(t)=j \mid \mathcal{M}\left(\tau_{0}^{*}\right)=i\right\} \\
& \mathbf{K}(t)=\left[K_{i j}(t)\right] \quad " \quad K_{i j}(t)=\operatorname{Pr}\left\{\mathcal{M}\left(\tau_{1}^{*}\right)=j, \tau_{1}^{*} \leq t \mid \mathcal{M}\left(\tau_{0}^{*}\right)=i\right\} \\
& \mathbf{E}(t)=\left[E_{i j}(t)\right] \quad " \quad E_{i j}(t)=\operatorname{Pr}\left\{\mathcal{M}(t)=j, \tau_{1}^{*}>t \mid \mathcal{M}\left(\tau_{0}^{*}\right)=i\right\}
\end{aligned}
$$

Matrix $\mathbf{V}(t)$ is the transition probability matrix and provides the probability that the stochastic process $\mathcal{M}(t)$ is in marking $j$ at time $t$ given it was in $i$ at $t=0$. The matrix $\mathbf{K}(t)$ is the global kernel of the MRGP and provides the cdf of the event that the next regeneration time point is $\tau_{1}^{*}$ and the next regeneration marking is $M_{1}=j$ given marking $i$ at $\tau_{0}^{*}=0$. Finally, the matrix $\mathbf{E}(t)$ is the local kernel since it describes the behavior of the marking process $\mathcal{M}(t)$ inside two consecutive regeneration time points. The generic element $E_{i j}(t)$ provides the probability that the process is found in state $j$ at time $t$ starting from $i$ at $\tau_{0}^{*}=0$ before the next regeneration time point. From the above definitions:

$$
\sum_{j}\left[K_{i j}(t)+E_{i j}(t)\right]=1
$$

The transient behavior of the $M R S P N$ can be evaluated by solving the following generalized Markov renewal equation (in matrix form) [10, 8]:

$$
\mathbf{V}(t)=\mathbf{E}(t)+\mathbf{K} * \mathbf{V}(t)
$$

where $\mathbf{K} * \mathbf{V}(t)$ is a convolution matrix, whose $(i, j)$-th entry is:

$$
[\mathbf{K} * \mathbf{V}(t)]_{i j}=\sum_{k} \int_{0}^{t} d K_{i k}(y) V_{k j}(t-y)
$$

By denoting the Laplace Stieltjes transform $(L S T)$ of a function $F(t)$ by $F^{\sim}(s)=\int_{0}^{\infty} e^{-s t} d F(t)$, Equation (3) becomes in the LST domain:

$$
\mathbf{V}^{\sim}(s)=\mathbf{E}^{\sim}(s)+\mathbf{K}^{\sim}(s) \mathbf{V}^{\sim}(s)
$$

whose solution is:

$$
\mathbf{V}^{\sim}(s)=\left[\mathbf{I}-\mathbf{K}^{\sim}(s)\right]^{-1} \mathbf{E}^{\sim}(s)
$$

The steady state solution can be evaluated as:

$$
P_{i j}=\lim _{t \rightarrow \infty} \operatorname{Pr}\{\mathcal{M}(t)=j \mid \mathcal{M}(0)=i\}=\lim _{t \rightarrow \infty} V_{i j}(t)=\lim _{s \rightarrow 0} V_{i j}^{\sim}(s)
$$

As specified by (2), $\mathbf{K}(t)$ and $\mathbf{E}(t)$ depend on the evolution of the marking process between two consecutive regeneration time points. By virtue of the time homogeneity property (1), we can always define the two successive regeneration time points to be $t=\tau_{0}^{*}=0$ and $t=\tau_{1}^{*}$. 
Definition 3 - The stochastic process subordinated to state $i$ (denoted by $\mathcal{M}^{i}(t)$ ) is the restriction of the marking process $\mathcal{M}(t)$ for $t \leq \tau_{1}^{*}$ given $\mathcal{M}\left(\tau_{0}^{*}\right)=i$ :

$$
\mathcal{M}^{i}(t)=\left[\mathcal{M}(t): t \leq \tau_{1}^{*}, \mathcal{M}\left(\tau_{0}^{*}\right)=i\right]
$$

According to Definition $3, \mathcal{M}^{i}(t)$ describes the evolution of the $P N$ starting at the regeneration time point $\tau^{*}=0$ in the marking $i$, up to the next regeneration time point $\tau_{1}^{*}$. Therefore, $\mathcal{M}^{i}(t)$ includes all the markings that can be reached from state $i$ before the next regeneration time point. The entries of the $i$-th row of the matrices $\mathbf{K}(t)$ and $\mathbf{E}(t)$ are determined by $\mathcal{M}^{i}(t)$.

\section{MRSPN with non-overlapping activity cycles}

In order to restrict Definition 2 to a class of solvable models, the concept of MRSPN with non overlapping activity cycles has been introduced in [6]. This new class encompasses and generalizes all the models previously published in the literature and mentioned in the previous sections.

Definition 4 - A GEN transition is dormant in those markings in which the corresponding age variable is equal to zero and is active in those markings in which the age variable is greater than zero. The activity cycle of a GEN transition is the period of time in which a transition is active between two dormant periods.

Let $t r_{g}$ be a GEN transition. The activity cycle of $t r_{g}$ is influenced by its memory policy, and can be characterized in the following way:

- Resampling Memory - If $t r_{g}$ is a resampling memory transition, its activity cycle starts as soon as $t r_{g}$ becomes enabled, and ends at the first subsequent firing of any transition (including $t r_{g}$ itself). During the activity cycle of a resampling memory transition no change of marking is possible.

- Enabling Memory - If $t r_{g}$ is an enabling memory transition its activity cycle starts as soon as $t r_{g}$ becomes enabled when dormant, and ends either when $t r_{g}$ fires, or when it becomes disabled by the firing of a competitive transition. During the activity cycle the marking can change inside the subset of connected markings in which $t r_{g}$ is enabled. The age variable associated to $t r_{g}$ grows continuously during the activity cycle starting from 0 .

- Age Memory - If $t r_{g}$ is an age memory transition, its activity cycle starts as soon as $t r_{g}$ becomes enabled when dormant, and ends only at the firing of $t r_{g}$ itself. During the activity cycle of an age memory transition there is no restriction on the markings reachable by the marking process. The age memory policy is the only policy in which a transition can be active even in markings in which is not enabled. During the activity cycle, the age variable is non-decreasing in the sense that increases continuously in those markings in which $t r_{g}$ is enabled and maintains its constant positive value in those markings in which $t r_{g}$ is not enabled. The enabling/disabling condition of $t r_{g}$ during its activity cycle is tracked by introducing a reward (indicator) variable which is set to 1 in those markings in which $t r_{g}$ is enabled and set to 0 in those markings in which $t r_{g}$ is not enabled. With this assignment, the value of the age variable versus time can be computed as the total accumulated reward.

Definition 5 - Activity cycles are non-overlapping if there exists a dominant transition whose activity cycle strictly contains the activity cycles of all the active transitions. 
Definition 6 - A MRSPN with non-overlapping activity cycles is a MRSPN in which all the regeneration periods are dominated by a single transition: any two successive regeneration time points correspond to the start and to the end of the activity cycle of the dominant transition.

Definition 6, includes the possibility that the activity cycles of GEN transitions are completely contained into the activity cycle of the dominant one, hence allowing the simultaneous enabling of different GEN transitions inside the same subordinated process. In particular in [6], the class of models for which the process subordinated to any GEN transition is a semi Markov reward process is defined and analyzed. In order to track the enabling/disabling condition of the dominant GEN transition $t r_{g}$, a reward is introduced which assumes the following values:

- 1 if $t r_{g}$ is enabled in the given marking;

- 0 if $t r_{g}$ is not enabled.

The binary reward variables can then be grouped into a reward vector $\mathbf{r}$, which provides the state of each timed transition (enabled/disabled) in a given marking. In the remaining part of the paper we concentrate our analysis to the particular case of a $M R S P N$ in which the dominant transition can be GEN and either with associated enabling or age memory policy, but with $C T M C$ subordinated processes.

\section{Unified transient analysis of MRSPN}

In this Section, we briefly summarize the theory for the transient analysis of MRSPN with non-overlapping activity cycles and subordinated CTMC. An extension to subordinated semiMarkov processes and major details on the analytical procedure can be found in $[6,18]$. In this section we show how to derive row by row the global kernel $\mathbf{K}(t)$ and the local kernel $\mathbf{E}(t)$. Hence, we assume to start a new regeneration period at time $t=0$ in marking $i$, being $\operatorname{tr}_{g}$ the dominant GEN transition and being the subordinated process a reward $C T M C$. To better understand the developed mathematical formalism, we briefly describe the adopted notation:

- $\Omega$ : reachability set $\mathcal{R S}\left(M_{0}\right)$;

- $n$ : cardinality of the reachability set;

- r: vector of the reward variables associated to each timed transition in a given state;

- $R^{i}$ : subset of $\Omega$ grouping the states reachable from state $i$ before firing $\operatorname{tr}_{g}$ (the associate age variable is $\neq 0$ );

- $m$ : cardinality of $R^{i}$;

- $h$ : cardinality of the subset of $R^{i}$ in which the age variable associated with $t r_{g}$ is stricly increasing (the reward rate is equal to 1 );

- $R^{c i}$ : subset of $\Omega$ in which $t r_{g}$ is not active. Namely, $R^{c i}=\Omega-R^{i}$;

- $Z^{i}(t)$ : subordinated $C T M C$ defined over $R^{i}$;

- $\mathrm{A}^{i}=\left[a_{k \ell}^{i}\right]$ : generator matrix of the subordinated $C T M C Z^{i}(t)$. 
Let us renumber the states in $\mathbf{A}^{i}$ in such a way that states numbered $1,2, \ldots, m$ belong to $R^{i}$ $\left(1,2, \ldots, m \in R^{i}\right)$ and the states numbered $m+1, m+2, \ldots, n$ belong to $R^{c i}(m+1, m+2, \ldots, n \in$ $R^{c i}$ ). By this ordering of states $\mathbf{A}^{i}$ can be partitioned into the following submatrices $\mathbf{A}^{i}=$ \begin{tabular}{|c|c|}
\hline $\mathbf{B}$ & $\mathbf{C}$ \\
\hline $\mathbf{0}$ & $\mathbf{0}$ \\
\hline
\end{tabular} where $\mathbf{B}$ contains the intensity of the transitions inside $R^{i}$, and $\mathbf{C}$ contains the intensity of the transitions from $R^{i}$ to $R^{c i}$, being the other submatrices irrelevant.

Fix the value of the firing requirement $\gamma_{g}=w$, and define the following matrix functions $\mathbf{P}^{i}(t, w), \mathbf{F}^{i}(t, w), \mathbf{D}^{i}(t, w)$ and $\boldsymbol{\Delta}^{i}$ :

$$
\begin{gathered}
P_{k \ell}^{i}(t, w)=\operatorname{Pr}\left\{Z^{i}(t)=\ell \in R^{i}, \tau_{1}^{*}>t \mid Z^{i}(0)=k \in R^{i}, \gamma_{g}=w\right\} \\
F_{k \ell}^{i}(t, w)=\operatorname{Pr}\left\{Z^{i}\left(\tau_{1}^{*-}\right)=\ell \in R^{i}, \tau_{1}^{*} \leq t, t r_{g} \text { fires } \mid Z^{i}(0)=k \in R^{i}, \gamma_{g}=w\right\} \\
D_{k \ell}^{i}(t, w)=\operatorname{Pr}\left\{Z^{i}\left(\tau_{1}^{*}\right)=\ell \in R^{c i}, \tau_{1}^{*} \leq t \mid Z^{i}(0)=k \in R^{i}, \gamma_{g}=w\right\} \\
\Delta_{k \ell}^{i}=\operatorname{Pr}\left\{\text { next tangible marking is } \ell \mid \text { current marking is } k, t r_{g} \text { fires }\right\}
\end{gathered}
$$

By the above definitions, $P_{k \ell}^{i}(t, w)$ and $F_{k \ell}^{i}(t, w)$ are 0 if $k \in \Omega-R^{i}$ or $\ell \in \Omega-R^{i}$; and $D_{k \ell}^{i}(t, w)$ is 0 if $k \in \Omega-R^{i}$ or $\ell \in R^{i}$.

- $P_{k \ell}^{i}(t, w)$ is the probability of being in state $\ell \in R^{i}$ at time $t$ before absorption either at the barrier $w$ or in the absorbing subset $R^{c i}$, starting in state $k \in R^{i}$ at $t=0$.

- $F_{k \ell}^{i}(t, w)$ is the probability that $t r_{g}$ fires from state $\ell \in R^{i}$ (hitting the absorbing barrier $w$ in $\ell$ ) before $t$, starting in state $k \in R^{i}$ at $t=0$.

- $D_{k \ell}^{i}(t, w)$ is the probability of first passage from a state $k \in R^{i}$ to a state $\ell \in R^{c i}$ before hitting the barrier $w$, starting in state $k \in R^{i}$ at $t=0$.

- $\boldsymbol{\Delta}^{i}$ is the branching probability matrix and represents the successor tangible marking $\ell$ that is reached by firing $t r_{g}$ in state $k \in R^{i}$ (the firing of $t r_{g}$ in the subordinated process $\mathcal{M}^{i}(t)$, can only occur in a state $k$ in which $\left.r_{k}^{i}=1\right)$.

From (8), (9) and (10), it follows for any $t$ :

$$
\sum_{\ell \in \Omega^{i}}\left[P_{k \ell}^{i}(t, w)+F_{k \ell}^{i}(t, w)+D_{k \ell}^{i}(t, w)\right]=1
$$

Given that $G_{g}(w)$ is the cumulative distribution function of the r.v. $\gamma_{g}$ associated to the transition $t r_{g}$, the elements of the $i$-th row of matrices $\mathbf{K}(t)$ and $\mathbf{E}(t)$ can be expressed as follows, as a function of the matrices $\mathbf{P}^{i}(t, w), \mathbf{F}^{i}(t, w)$ and $\mathbf{D}^{i}(t, w)$ :

$$
\begin{gathered}
K_{i j}(t)=\int_{w=0}^{\infty}\left[\sum_{k \in R^{i}} F_{i k}^{i}(t, w) \Delta_{k j}^{i}+D_{i j}^{i}(t, w)\right] d G_{g}(w) \\
E_{i j}(t)=\int_{w=0}^{\infty} P_{i j}^{i}(t, w) d G_{g}(w)
\end{gathered}
$$

In order to avoid unnecessarily cumbersome notation in the following of this section, we neglect the explicit dependence on the particular subordinated process $Z^{i}(t)$, by eliminating the superscript $i$. It is however tacitly intended, that all the quantities $\mathbf{r}, \mathbf{A}, \mathbf{P}(t, w), \mathbf{F}(t, w), \mathbf{D}(t, w)$, $\Delta, R$ and $R^{c}$ refer to the specific process subordinated to the regeneration period starting from state $i$. 
The introduced matrix functions are derived in double Laplace transform domain based on the infinitesimal generator of the subordinated $C T M C$ A and on the memory policy of the dominant GEN transition. A detailed derivation is in $[18,5]$. The final expressions take the following form.

$$
\begin{aligned}
& \left(s+v r_{k}\right) F_{k \ell}^{\sim *}(s, v)=\delta_{k \ell} r_{k}+\sum_{u \in R} a_{k u} F_{u \ell}^{\sim *}(s, v) \\
& \left(s+v r_{k}\right) P_{k \ell}^{\sim *}(s, v)=\delta_{k \ell} \frac{s}{v}+\sum_{u \in R} a_{k u} P_{u \ell}^{\sim *}(s, v) \\
& \left(s+v r_{k}\right) D_{k \ell}^{\sim *}(s, v)=\frac{a_{k \ell}}{v}+\sum_{u \in R} a_{k u} D_{u \ell}^{\sim *}(s, v)
\end{aligned}
$$

Equations (13), (12) and (14) can be rewritten in matrix form:

$$
\begin{aligned}
\mathbf{F}^{\sim *}(s, v) & =(s \mathbf{I}+v \mathbf{R}-\mathbf{B})^{-1} \mathbf{R} \\
\mathbf{P}^{\sim *}(s, v) & =\frac{s}{v}(s \mathbf{I}+v \mathbf{R}-\mathbf{B})^{-1} \\
\mathbf{D}^{\sim *}(s, v) & =\frac{1}{v}(s \mathbf{I}+v \mathbf{R}-\mathbf{B})^{-1} \mathbf{C}
\end{aligned}
$$

where $\mathbf{I}$ is the identity matrix and $\mathbf{R}$ is the diagonal matrix of the reward rates $\left(r_{k}\right)$; the dimensions of $\mathbf{I}, \mathbf{R}, \mathbf{B}, \mathbf{F}$ and $\mathbf{P}$ are $(m \times m)$, and the dimensions of $\mathbf{C}$ and $\mathbf{D}$ are $(m \times(n-m))$.

Starting from Equations (12-17), a natural way to evaluate the steady state probabilities of a MRSPN goes through the following steps:

1. generation of the reachability tree and identification of the subordinated processes;

2. derivation of the entries of the $\mathbf{P}^{* \sim}(s, v), \mathbf{F}^{* \sim}(s, v)$ and $\mathbf{D}^{* \sim}(s, v)$ matrices symbolically in the double Laplace transform domain for the different subordinated processes by (15), (16) and (17);

3. symbolical inverse Laplace transformation of $\mathbf{P}^{* \sim}(s, v), \mathbf{F}^{* \sim}(s, v)$ and $\mathbf{D}^{* \sim}(s, v)$ with respect to $v$;

4. unconditioning of the entries of the $\mathbf{K}^{\sim}(s)$ and $\mathbf{E}^{\sim}(s)$ matrices, according to the $\mathrm{Cdf}$ of the GEN distributions (11).

5. symbolical matrix inversion and matrix multiplication by using a standard package in order to obtain the $\mathbf{V}^{\sim}(s)$ matrix (6) in the LT domain;

6. evaluation the limiting behaviour by ( 7$)$.

But this way of the analysis contains some computationally intensive steps, and the whole procedure can be applied to very small scale problems (5-8 states) only.

\section{$5 \quad$ Steady state analysis of MRSPN with subordinated CTMC}

We propose a new, computationally effective, approach based on the procedure published by Ajmone Marsan and Chiola [3]. The procedure is extended for the first time to GEN transitions with age memory policy. Let us define:

$$
\alpha_{i j}=\int_{t=0}^{\infty} E_{i j}(t) d t \quad ; \quad \alpha_{i}=\sum_{j} \alpha_{i j}
$$


the expected time the $Z^{i}(t)$ subordinated process spends in state $j$, and the expected duration of the activity cycle of transition $t r_{g}$.

The state transition probability matrix of the $D T M C$ embedded into the regeneration time points is

$$
\Pi=\left\{\pi_{i j}\right\}=\lim _{t \rightarrow \infty} \mathbf{K}(t)
$$

Let $P=\left\{p_{i}\right\}$ (row vector) be the unique solution of the set of equations:

$$
P=P \Pi \quad ; \quad \sum_{i} p_{i}=1
$$

The steady state probabilities of the $M R G P$ can be evaluated based on $\alpha_{i j}$ and $\pi_{i j}$ (or $p_{i}$ by applying (20)) as follows [3]:

$$
v_{i j}=\lim _{t \rightarrow \infty} \operatorname{Pr}\{\mathcal{M}(t)=j \mid \mathcal{M}(0)=i\}=\frac{\sum_{k} p_{k} \alpha_{k j}}{\sum_{k} p_{k} \alpha_{k}}
$$

Thus, the steady state solution can be easily obtained once the time conversion factor $\alpha_{i j}$ and the state transition probabilities $\pi_{i j}$ of the DTMC embedded into the regeneration points are known. Obviously, the memory policy for GEN transitions is taken into account in the evaluation of the local and global kernels.

In the following two Subsections we evaluate the analytical expression of both $\alpha_{i j}$ and $\pi_{i j}$. The particular expressions for the enabling and age memory cases are derived in the Section 6 .

\subsection{Time conversion factor $\alpha_{i j}$}

Let us suppose that the states of $R^{i}$ in which the dominant transition is enabled (reward rate $=1)$ are numbered as $1,2, \ldots, h(h \leq m)$ and the states of $R^{i}$ in which the dominant transition is disabled (reward rate $=0$ ) are numbered $h+1, h+2, \ldots, m$. By this ordering of the infinitesimal generator $\mathbf{A}^{\mathrm{i}}$ of the subordinated $C T M C$ starting from state $i$ can be partitioned into the following submatrices

$$
\mathbf{A}^{\mathbf{i}}=\begin{array}{|c|c|}
\hline \mathbf{B} & \mathbf{C} \\
\hline \mathbf{0} & \mathbf{0} \\
\hline
\end{array}=\begin{array}{|l|l|l|}
\hline B_{1} & B_{2} & \mathbf{C} \\
\hline B_{3} & B_{4} & \\
\hline \mathbf{0} & \mathbf{0} \\
\hline
\end{array}
$$

In the notation of the particular submatrices we neglect the dependence on the particular subordinated process, and superscript $i$ is omitted.

Theorem 1 The expected time the subordinated CTMC $Z^{i}(t)$ spends in state $j$ is:

$$
\alpha_{i j}^{i}=\int_{w=0}^{\infty}\left[\begin{array}{cc}
L(w) & -L(w) \mathrm{B}_{2} \mathrm{~B}_{4}{ }^{-1} \\
-\mathrm{B}_{4}{ }^{-1} \mathrm{~B}_{3} L(w) & \mathrm{B}_{4}{ }^{-1}+\mathrm{B}_{4}{ }^{-1} \mathrm{~B}_{3} L(w) \mathrm{B}_{2} \mathrm{~B}_{4}{ }^{-1}
\end{array}\right]_{i j} d G_{g}(w)
$$

where

$$
\beta=\mathrm{B}_{1}-\mathrm{B}_{2} \mathrm{~B}_{4}{ }^{-1} \mathrm{~B}_{3} \quad, \quad L(w)=\int_{0}^{w} e^{\beta w} d w .
$$

and superscript $i$ denotes the fact that the states are rearranged according to the regeneration period starting from state $i$, and that the equation holds only for the ith row of the matrices. 
Proof:

$$
\begin{aligned}
& \alpha_{i j}^{i}=\int_{t=0}^{\infty} E_{i j}(t) d t=\lim _{s \rightarrow 0} \frac{1}{s} E_{i j}^{\sim}(s)= \\
& \lim _{s \rightarrow 0} \frac{1}{s} \int_{w=0}^{\infty} P_{i j}^{i} \sim(s, w) d G_{g}(w)= \\
& \int_{w=0}^{\infty} \operatorname{LT}^{-1}\left[\frac{1}{v}\left|(v \mathbf{R}-\mathbf{B})^{-1}\right|_{i j}\right] d G_{g}(w)
\end{aligned}
$$

Let us consider the term $\mathrm{LT}^{-1}\left[\frac{1}{v}(v \mathbf{R}-\mathbf{B})^{-1}\right]$ separately. Based on the numbering of the states $\mathbf{R}$ has the form $\mathbf{R}=$\begin{tabular}{|c|c|}
\hline$I_{1}$ & 0 \\
\hline 0 & 0 \\
\hline
\end{tabular}$\quad$ where $\mathrm{I}_{1}$ is the identity matrix of dimension $(h \times h)$. Using this and Lemma 1 in the Appendix, the inverse Laplace transform satisfies the following equation:

$$
\begin{aligned}
& \mathrm{LT}^{-1}\left[\frac{1}{v}(v \mathbf{R}-\mathbf{B})^{-1}\right]=\mathrm{LT}^{-1}\left\{\frac{1}{v}\left[\begin{array}{cc}
v \mathrm{I}_{1}-\mathrm{B}_{1} & -\mathrm{B}_{2} \\
-\mathrm{B}_{3} & -\mathrm{B}_{4}
\end{array}\right]^{-1}\right\}= \\
& \mathrm{LT}^{-1}\left\{\frac{1}{v}\left[\begin{array}{cc}
\left(v \mathrm{I}_{1}-\beta\right)^{-1} & -\left(v \mathrm{I}_{1}-\beta\right)^{-1} \mathrm{~B}_{2} \mathrm{~B}_{4}^{-1} \\
-\mathrm{B}_{4}^{-1} \mathrm{~B}_{3}\left(v \mathrm{I}_{1}-\beta\right)^{-1} & \mathrm{~B}_{4}^{-1}+\mathrm{B}_{4}^{-1} \mathrm{~B}_{3}\left(v \mathrm{I}_{1}-\beta\right)^{-1} \mathrm{~B}_{2} \mathrm{~B}_{4}^{-1}
\end{array}\right]\right\}= \\
& {\left[\begin{array}{cc}
L(w) & -L(w) \mathrm{B}_{2} \mathrm{~B}_{4}{ }^{-1} \\
-\mathrm{B}_{4}{ }^{-1} \mathrm{~B}_{3} L(w) & \mathbf{B}_{4}{ }^{-1}+\mathrm{B}_{4}{ }^{-1} \mathrm{~B}_{3} L(w) \mathbf{B}_{2} \mathbf{B}_{4}{ }^{-1}
\end{array}\right]}
\end{aligned}
$$

where $L(w)=\int_{0}^{w} e^{\beta w} d w$ (see Lemma 3 in the Appendix). From this the theorem follows.

\subsection{State transition probabilities of the EMC $\pi_{i j}$}

Theorem 2 The state transition probabilities of the DTMC embedded into the regeneration time points are given by:

$$
\begin{aligned}
& \pi_{i j}^{i}=\int_{w=0}^{\infty} \sum_{k \leq h}\left[\begin{array}{cc}
e^{\beta w} & 0 \\
-\mathrm{B}_{4}{ }^{-1} \mathrm{~B}_{3} e^{\beta w} & 0
\end{array}\right]_{i k} \Delta_{k j}^{i} d G_{g}(w) \\
& +\int_{w=0}^{\infty} \sum_{k \leq m}\left[\begin{array}{cc}
L(w) & -L(w) \mathrm{B}_{2} \mathrm{~B}_{4}^{-1} \\
-\mathrm{B}_{4}{ }^{-1} \mathrm{~B}_{3} L(w) & \mathrm{B}_{4}^{-1}+\mathrm{B}_{4}^{-1} \mathrm{~B}_{3} L(w) \mathrm{B}_{2} \mathrm{~B}_{4}^{-1}
\end{array}\right]_{i k} C_{k j} d G_{g}(w)
\end{aligned}
$$

\section{Proof:}




$$
\begin{aligned}
& \pi_{i j}^{i}=\lim _{t \rightarrow \infty} K_{i j}(t)=\lim _{s \rightarrow 0} K_{i j}^{\sim}(s)= \\
& \lim _{s \rightarrow 0} \int_{w=0}^{\infty} \sum_{k \leq h} F_{i k}^{i \sim}(s, w) \Delta_{k j}^{i}+D_{i j}^{i} \sim(s, w) d G_{g}(w)= \\
& \int_{w=0}^{\infty} \sum_{k \leq h} \mathrm{LT}^{-1}\left[(v \mathbf{R}-\mathbf{B})^{-1} \mathbf{R}\right]_{i k} \Delta_{k j}^{i} d G_{g}(w) \\
& +\int_{w=0}^{\infty} \sum_{k \leq m} \operatorname{LT}^{-1}\left[\frac{1}{v}(v \mathbf{R}-\mathbf{B})^{-1}\right]_{i k} C_{k j} d G_{g}(w)
\end{aligned}
$$

Let us consider the term $\mathrm{LT}^{-1}\left[(v \mathbf{R}-\mathbf{B})^{-1} \mathbf{R}\right]$ separately. By the partitioned form of $\mathbf{R}$ and $\mathbf{B}$ and adopting Lemma 2 in the Appendix, the inverse Laplace transform satisfies the following equation:

$$
\begin{aligned}
& \mathrm{LT}^{-1}\left[(v \mathbf{R}-\mathbf{B})^{-1} \mathbf{R}\right]=\mathrm{LT}^{-1}\left\{\left[\begin{array}{cc}
v \mathrm{I}_{1}-\mathrm{B}_{1} & -\mathrm{B}_{2} \\
-\mathrm{B}_{3} & -\mathrm{B}_{4}
\end{array}\right]^{-1} \mathbf{R}\right\}= \\
& \mathrm{LT}^{-1}\left[\begin{array}{cc}
\left(v \mathrm{I}_{1}-\beta\right)^{-1} & 0 \\
-\mathrm{B}_{4}^{-1} \mathrm{~B}_{3}\left(v \mathrm{I}_{1}-\beta\right)^{-1} & 0
\end{array}\right]=\left[\begin{array}{cc}
e^{\beta w} & 0 \\
-\mathrm{B}_{4}^{-1} \mathrm{~B}_{3} e^{\beta w} & 0
\end{array}\right]
\end{aligned}
$$

The term $\mathrm{LT}^{-1}\left[\frac{1}{v}(v \mathbf{R}-\mathbf{B})^{-1}\right]$ is known from $(24)$.

\section{Computational method}

The relevant entries of the matrices $\left(\alpha_{i j}^{i}, \pi_{i j}^{i}\right)$ are located in row $i(i \leq h)$, because when the subordinated process of the dominant transition $t r_{g}$ starts, transition $t r_{g}$ is enabled. The expressions for $\alpha_{i j}^{i}$ and $\pi_{i j}^{i}$ are evaluated in the following subsections assuming enabling and age type memory policy, respectively. The following subsections provide the final results, under the assumption that the subortinated process is a reward $C T M C$.

\subsection{Enabling type dominant transition}

In the case of enabling type dominant transition $h=m$ and $m<n$, hence $\beta=\mathrm{B}_{1}$ and the partitioned form of the infinitesimal generator of the subordinated process is $\mathbf{A}^{\mathbf{i}}=$\begin{tabular}{|c|c|}
\hline $\mathrm{B}_{1}$ & $\mathbf{C}$ \\
\hline $\mathbf{0}$ & $\mathbf{0}$ \\
\hline
\end{tabular} Note that $\mathbf{A}^{\mathbf{i}}$ is a $(n \times n)$ matrix, and, in this case, $\mathbf{B}_{1}$ is a $(h \times h)$ matrix.

$$
\begin{gathered}
\alpha_{i j}^{i}=\int_{w=0}^{\infty}[L(w)]_{i j} d G_{g}(w)=\int_{w=0}^{\infty} \int_{\tau=0}^{w}\left[e^{\tau \mathrm{B}_{1}}\right]_{i j} d \tau d G_{g}(w) \\
\pi_{i j}^{i}=\int_{w=0}^{\infty} \sum_{k \leq h}\left[e^{\mathrm{B}_{1} w}\right]_{i k} \Delta_{k j}^{i} d G_{g}(w)+\int_{w=0}^{\infty} \sum_{k \leq m}[L(w)]_{i k} C_{k j} d G_{g}(w)
\end{gathered}
$$




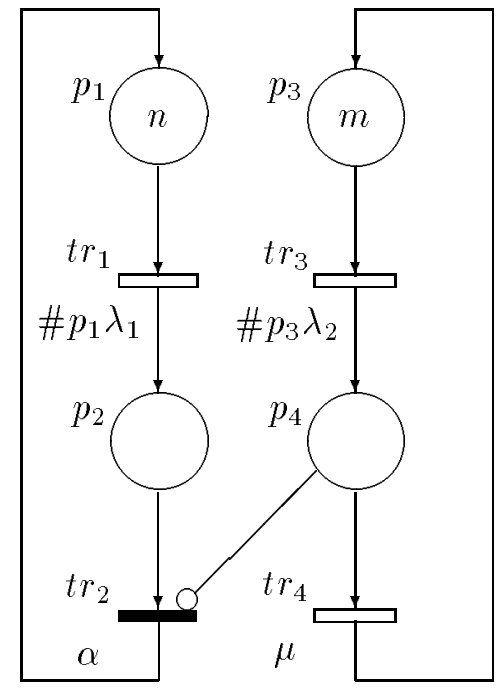

Figure 1 - Preemptive M/D $/ 1 / n+m / n+m$ queue with two classes of customers.

As can be observed, in evaluating $e^{A^{i} t}$ we just need to concentrate on the submatrix $B_{1}$. The complexity of the proposed algorithm is thus in the evaluation of $e^{\mathrm{B}_{1} t}$. This observation allows us to save computational time. Other algorithms, such as those proposed in [16, 8], evaluate $e^{A^{i} t}$, even if it is not the case.

\subsection{Age type dominant transition}

In the case of age type dominant transition $h \leq m$ and $m=n$, hence the partitioned form of the infinitesimal generator of the subordinated process is $\mathbf{A}^{\mathbf{i}}=$\begin{tabular}{|l|l|}
\hline$B_{1}$ & $B_{2}$ \\
\hline$B_{3}$ & $B_{4}$ \\
\hline
\end{tabular} and $D_{i j}^{i} \sim(s, w)=0$.

$$
\begin{gathered}
\alpha_{i j}^{i}=\int_{w=0}^{\infty}\left[L(w) \mid-L(w) \mathrm{B}_{2} \mathrm{~B}_{4}{ }^{-1}\right]_{i j} d G_{g}(w) \\
\pi_{i j}^{i}=\int_{w=0}^{\infty} \sum_{k \leq h}\left[e^{\beta w}\right]_{i k} \Delta_{k j}^{i} d G_{g}(w)
\end{gathered}
$$

The complexity of the algorithm for the age type dominant transition, is related to the evaluation of $e^{\beta t}$, where $\beta$ is a $(h \times h)$ matrix, and to the evaluation of the inverse of matrix $B_{4}$ which is an $(m-h \times m-h)$ matrix.

\section{Numerical example: Preemptive $\mathbf{M} / \mathbf{D} / \mathbf{1} / n+m / n+m$ with two classes of customers}

The $P N$ of Figure 1 models a M/D $/ 1 / n+m / n+m$ queue in which the jobs submitted by customer 2 have higher priority and preempt the jobs submitted by customer 1 . This model, with $n=m=1$, has been addressed for the first time in [5] where the service times were assumed to be both deterministic with age memory policy.

Here, we generalize the original model to any number of customers: the requested service time of customer 2 is exponentially distributed, but it is deterministic for customer 1 . The 

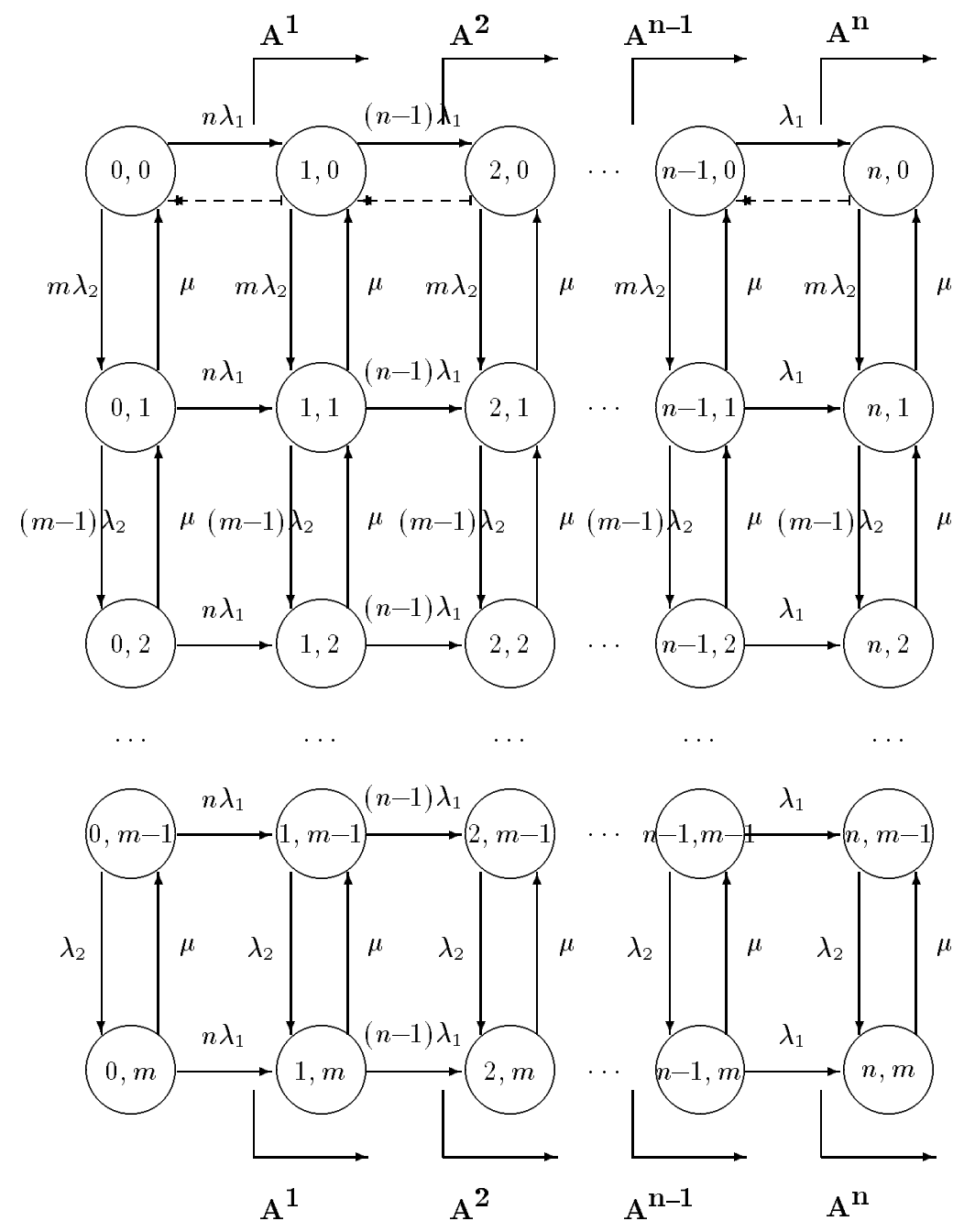

Figure 2 - State transition diagram of the preemptive M/D/1/n+m/n+m queue with two classes of customers.

number of tokens in place $p_{1}\left(p_{3}\right)$ represents the number of customers of type 1 (type 2) thinking. Tokens in place $p_{2}\left(p_{4}\right)$ represent the numebr of jobs of type 1 (type 2) queueing for service (included the one under service). Transitions $t r_{1}$ and $t r_{3}$ are EXP and represent the submission of a job of type 1 or 2, respectively. $t r_{2}$ is deterministic and $t r_{4}$ is EXP transition, and represent the completion of service of a job of type 1 or 2, respectively. A prs service discipline is modeled by assigning to $t r_{2}$ an age memory policy. Under a prs service, after completion of the type 2 job, the interrupted type 1 job is resumed continuing the new service period from the point reached just before the last interruption. The inhibitor arc from $p_{4}$ to $t r_{2}$ models the described preemption mechanism: as soon as a type 2 job joins the queue the type 1 job eventually under service is interrupted.

The reachability graph of the $P N$ of Figure 1 is depicted in Figure 2. States in Figure 2 are labeled with two indices: the first index is the number of tokens in place $p_{2}\left(\# p_{2}\right)$, the second index is the number of tokens in place $p_{4}\left(\# p_{4}\right)$. Let us define a numbering of the states between 1 and $(n+1) \times(m+1)$ based on the following rule: $i=1+\# p_{2}+\# p_{4} \times(n+1)$. For 
notational convenience we denote $[(i-1) \bmod (n+1)]$ by $i^{*}$ and $[(i-1)$ div $(n+1)]$ by $i^{+}$.

From Figure 2, it is easily recognized that all states can be regeneration states. Only exponential transitions are enabled in state 1 and states $\{i: i>n+1\}$. The elements of the $\Pi$ and $\alpha$ matrices can be easily defined for these states:

for $i=1$ and $i>n+1$

$$
\pi_{i j}=\left\{\begin{array}{cc}
\frac{\left(n-i^{*}\right) \lambda_{1}}{\left(n-i^{*}\right) \lambda_{1}+\left(m-i^{+}\right) \lambda_{2}+\mu} & j=i+1 \\
\frac{\left(m-i^{+}\right) \lambda_{2}}{\left(n-i^{*}\right) \lambda_{1}+\left(m-i^{+}\right) \lambda_{2}+\mu} & j=i+n+1 \\
\frac{\mu}{\left(n-i^{*}\right) \lambda_{1}+\left(m-i^{+}\right) \lambda_{2}+\mu} & \text { otherwise } \\
0 & \\
\alpha_{i i}=\alpha_{i}=\frac{\alpha_{i j}=0 \text { if } j \neq i}{\left(n-i^{*}\right) \lambda_{1}+\left(m-i^{+}\right) \lambda_{2}+\mu} & ;
\end{array}\right.
$$

States $\{i: 1<i \leq n+1\}$ are age type regeneration states. The state space of the subordinated process $R^{i}$ contains all the states reachable during the activity cycle of $t r_{2}$. The only criterion for the termination of the activity cycle is the firing of $t r_{2}$. The reward vector is equal to 1 for the states $j \in R^{i}$ in which $t r_{2}$ is enabled (states located in the first row on Figure 2 ) and 0 for the states $j \in R^{i}$ in which $t r_{2}$ is not enabled. As during the activity cycle of $t r_{g}$ no other GEN transitions are activated, the subordinated process is a reward CTMC. Then we meet the structural conditions required by the steady state solution method described in the previous Sections.

The reachable states and the subordinated $C T M C$ for state $i$ is denoted by $\mathrm{A}^{\mathrm{i}}$ in Figure 2 . (The deterministic state transitions /dashed lines/ should be excluded.) Every relevant row of the branching probability matrix contains only one non-zero entry $\Delta_{i, i-1}=1$.

Table 1 reports the steady-state results of the example of Figure 1 for a deterministic service duration $\alpha=1$, an exponential service duration of mean $\mu=1$, submitting rates $\lambda_{1}=\lambda_{2}=0.25$ and for $n=3, m=2$. The third column shows the results of the proposed method obtained through a MATHEMATICA implementation. Columns 4 and 5 provide the results obtained by approximating the deterministic service time $t r_{2}$ by an Erlang distribution of order 10 and 1000 respectively, and evaluating the resulting PHSPN model [4] utilizing the method in [12]. Column 6 contains the results for the same example solved by DSPNexpress [15], taking into account, however, that DSPNexpress supports enabling memory policy only. As can be observed, the proposed method provides the same accuracy of the Erlang approximation of order 1000 , but with a much more limited state space: 12 states against 9003 states. The results obtained by DSPNexpress are comparabale with caution, due to the different adopted memory policy. 


\begin{tabular}{||c|c||c||c|c||c||}
\hline \hline state & marking & $\begin{array}{c}\text { MRSPN method } \\
\text { age memory }\end{array}$ & $\begin{array}{c}\text { PHSPN(10) } \\
\text { age memory }\end{array}$ & $\begin{array}{c}\text { PHSPN(1000) } \\
\text { age memory }\end{array}$ & $\begin{array}{c}\text { DSPNexpress } \\
\text { enabling memory }\end{array}$ \\
\hline \hline & 3020 & 0.17960 & 0.18209 & 0.17963 & 0.13142 \\
2 & 2120 & 0.25286 & 0.24491 & 0.25278 & 0.22130 \\
3 & 1220 & 0.14814 & 0.14977 & 0.14815 & 0.18433 \\
4 & 0320 & 0.03478 & 0.03861 & 0.03482 & 0.07832 \\
5 & 3011 & 0.04835 & 0.04902 & 0.04836 & 0.03538 \\
6 & 2111 & 0.10493 & 0.10277 & 0.10491 & 0.08824 \\
7 & 1211 & 0.10378 & 0.10348 & 0.10378 & 0.11014 \\
8 & 0311 & 0.05061 & 0.05241 & 0.05064 & 0.07393 \\
9 & 3002 & 0.00691 & 0.00700 & 0.00691 & 0.00505 \\
10 & 2102 & 0.02094 & 0.02063 & 0.02094 & 0.01723 \\
11 & 1202 & 0.02913 & 0.02894 & 0.02913 & 0.02892 \\
12 & 0302 & 0.01994 & 0.02034 & 0.01994 & 0.02571 \\
\hline \hline
\end{tabular}

Table I - Steady state results of the preemptive M/D $/ 1 / n+m / n+m$ queue $(n=3, m=2)$

\section{Conclusion}

We considered GDT_SPN with age type memory policy. This kind of memory policy was motivated by the need of modeling systems in which the execution of tasks may follow a preemptive resume policy. We focused on the case in which the subordinated stochastic process is a CTMC and derived a computationally effective approach for the steady state analysis of Petri net with GEN transitions and associated age memory policy.

\section{Acknowledgements}

Kishor Trivedi and Andrea Bobbio would like to thank NATO for grant No. CRG.940308 Miklós Telek would like to thank Hungarian OTKA for grant No. W-015859. Antonio Puliafito would like to thank Italian CNR for grant No. 203.15.5.

\section{Appendix}

Lem ma 1 The inverse of the partitioned matrix $\mathcal{M}=$\begin{tabular}{|l|l|}
\hline $\mathcal{A}$ & $\mathcal{B}$ \\
\hline $\mathcal{C}$ & $\mathcal{D}$ \\
\hline
\end{tabular} has the following form, when $\operatorname{DET}(\mathcal{M}) \neq 0$ and $D E T(\mathcal{D}) \neq 0$ :

$$
\mathcal{N}=\left|\begin{array}{cc}
\left(\mathcal{A}-\mathcal{B D}^{-1} \mathcal{C}\right)^{-1} & -\left(\mathcal{A}-\mathcal{B D}^{-1} \mathcal{C}\right)^{-1} \mathcal{B D}^{-1} \\
-\mathcal{D}^{-1} \mathcal{C}\left(\mathcal{A}-\mathcal{B} \mathcal{D}^{-1} \mathcal{C}\right)^{-1} & \mathcal{D}^{-1}+\mathcal{D}^{-1} \mathcal{C}\left(\mathcal{A}-\mathcal{B D}^{-1} \mathcal{C}\right)^{-1} \mathcal{B D}^{-1}
\end{array}\right|
$$

Proof:

$$
\mathcal{N} \mathcal{M}=\mathbf{I} ; \quad \mathcal{M N}=\mathbf{I}
$$


Lem ma 2 Given matrix $A(n \times n)$, the inverse Laplace transform of $(s I-A)^{-1}$ has the following form:

$$
L T^{-1}(s I-A)^{-1}=e^{A t}
$$

Proof:

$$
\operatorname{LT}\left(e^{A t}\right)=(s I-A)^{-1}
$$

Lemma 3 Given matrix $A(n \times n)$, the inverse Laplace transform of $\frac{1}{s}(s I-A)^{-1}$ has the following form:

$$
L T^{-1}\left[\frac{1}{s}(s I-A)^{-1}\right]=\int_{0}^{\tau} e^{A t} d t=L(\tau)
$$

Proof: Given $Y(t)=\int_{0}^{t} F(\tau) d \tau$ by definition we have that:

$$
L T[Y(t)]=Y^{*}(s)=\frac{1}{s} F^{*}(s)
$$

where

$$
F^{*}(s)=L T[F(t)]
$$

If $F(t)=e^{A t}$ then:

$$
L T\left[\int_{0}^{t} e^{A \tau} d \tau\right]=\frac{1}{s} L T\left(e^{A \tau}\right)=\frac{1}{s}(s I-A)^{-1}
$$

From which the proof comes.

\section{References}

[1] M. Ajmone Marsan, G. Balbo, A. Bobbio, G. Chiola, G. Conte, and A. Cumani. The effect of execution policies on the semantics and analysis of stochastic Petri nets. IEEE Transactions on Software Engineering, SE-15:832-846, 1989.

[2] M. Ajmone Marsan, G. Balbo, and G. Conte. A class of generalized stochastic Petri nets for the performance evaluation of multiprocessor systems. ACM Transactions on Computer Systems, 2:93-122, 1984.

[3] M. Ajmone Marsan and G. Chiola. On Petri nets with deterministic and exponentially distributed firing times. In Lecture Notes in Computer Science, volume 266, pages 132-145. Springer Verlag, 1987.

[4] A. Bobbio and M. Telek. Computational restrictions for SPN with generally distributed transition times. In D. Hammer K. Echtle and D. Powell, editors, First European Dependable Computing Conference (EDCC-1), pages 131-148, 1994.

[5] A. Bobbio and M. Telek. Transient analysis of a preemptive resume M/D/1/2/2 through Petri nets. Technical report, Department of Telecommunications - Technical University of Budapest, April 1994. 
[6] A. Bobbio and M. Telek. Markov regenerative SPN with non-overlapping activity cycles. In International Computer Performance and Dependability Symposium - IPDS95, April 1995.

[7] V. Catania, A. Puliafito, M. Scarpa, and L. Vita. Concurrent generalized petri nets. In Proceedings of Numerical Solution of Markov Chains, pages 359-382, Raleigh, NC, 1995.

[8] Hoon Choi, V.G. Kulkarni, and K. Trivedi. Markov regenerative stochastic Petri nets. Performance Evaluation, 20:337-357, 1994.

[9] G. Ciardo, R. German, and C. Lindemann. A characterization of the stochastic process underlying a stochastic Petri net. IEEE Transactions on Software Engineering, 20:506$515,1994$.

[10] E. Cinlar. Introduction to Stochastic Processes. Prentice-Hall, Englewood Cliffs, 1975.

[11] D.R. Cox. The analysis of non-markovian stochastic processes by the inclusion of supplementary variables. Proceedings of the Cambridge Phylosophical Society, 51:433-440, 1955.

[12] A. Cumani. Esp - A package for the evaluation of stochastic Petri nets with phase-type distributed transition times. In Proceedings International Workshop Timed Petri Nets, pages 144-151, Torino (Italy), 1985. IEEE Computer Society Press no. 674.

[13] R. German. Transient Analysis of deterministic and stochastic Petri nets by the method of supplementary variables. Internal Report Technische Universität Berlin (to be presented MASCOT'95), 1994.

[14] R. German and C. Lindemann. Analysis of stochastic Petri nets by the method of supplementary variables. Performance Evaluation, 20:317-335, 1994.

[15] C. Lindemann. DSPNexpress: a software package for the efficient solution of deterministic and stochastic Petri nets. In R. Pooley and J. Hilliston, editors, Proceedings 6-th International Conference on Modelling Techniques and Tools for Computer Performance Evaluation, pages 15-29, Edinburgh, 1992.

[16] C. Lindemann. An improved numerical algorithm for calculating steady-state solutions of deterministic and stochastic Petri net models. Performance Evaluation, 18:75-95, 1993.

[17] D. Logothetis. Transient Analysis of Communication Networks. Ph.D. Thesis, Dept. of Electrical Eng., Duke University, 1994.

[18] Miklós Telek. Some advanced reliability modelling techniques. Phd Thesis, Hungarian Academy of Science, 1994. 